\title{
Lesiones macroscópicas en pulmones en ganado de lidia
}

\author{
A. Fernandez-Novo ${ }^{1, *}$, J.M. Lomillos-Pérez ${ }^{1}$ y J.A. García-García² \\ 1 Departamento de Producción y Sanidad Animal, Salud Pública Veterinaria y Ciencia y Tecnología de \\ los Alimentos, Universidad CEU Cardenal Herrera, C/ Tirant lo Blanc, 7. 46115 Alfara del Patriarca, \\ Valencia, España \\ 2 La Tejera SLP. C/ de la Cruz, 15. 28710 El Molar, Madrid, España
}

\section{Resumen}

El pulmón es el órgano encargado del intercambio gaseoso y de asegurar buena disponibilidad de oxígeno en situaciones de máximo ejercicio como es el espectáculo taurino. Por ello, presenta especial interés conocer cuáles son las principales afectaciones pulmonares vistas en canales post-mortem para así poder realizar mejoras a nivel productivo y de manejo, entre otras. En el presente trabajo se ha estudiado durante cinco años la aparición de lesiones pulmonares analizando 251 bovinos de lidia, evaluando la presencia de lesiones macroscópicas (enfisema, hemorragia, congestión y bronconeumonía) en pulmones de bovinos de diferentes edades y sexos. Metodología: se llevó a cabo en la comunidad de Madrid (Julio-Septiembre de los años 2013-2017). Resultados: $\mathrm{n}=251$ animales en los cinco años, 87/251 presentaron lesiones y alteraciones anatomopatológicas macroscópicas. Dentro de las lesiones y hallazgos agónicos observados: 22/87 correspondieron a enfisema pulmonar, 36/87 hemorragia, 24/87 congestión pulmonar, 4/87 bronconeumonía fibrinosa y 1/87 bronconeumonía supurativa. Los novillos (machos de dos-tres años) presentaron mayor frecuencia de lesiones $(44,30$ vs. 34,66\%; $P=0,013)$. La aparición de enfisema fue más frecuente en vacas que en otro tipo de animal $(53,33 \% ; P=0,023)$. La hemorragia apareció menos en vacas que en otro tipo de animal $(13,33 \% ; P=0,023)$, y tendencia a que apareciera menos en toro de capea $(P=0,023)$. El presente estudio ha revelado que dos de cada tres bóvidos de raza de lidia no presentan lesiones macroscópicas pulmonares, así como que la lesión agónica más frecuente fue la hemorragia pulmonar, seguida de congestión y enfisema sin observarse elevados porcentajes de bronconeumonía. Teniendo en cuenta que un $94,25 \%$ de los hallazgos observados corresponden a lesiones agónicas (presuntamente no correspondientes a patología previa incluyendo enfisema, hemorragia y congestión pulmonar), sólo un 5,75\% de las lesiones denotarían patología previa. Estos resultados podrían reflejar las condiciones de extensividad y máximos estándares de bienestar animal correspondientes a la tradición histórica de crianza del ganado bravo en dehesas.

Palabras clave: Espectáculo taurino, enfisema, hemorragia, bronconeumonía, post-mortem.

\section{Macroscopical lung lesions in bullfighting bovine}

\section{Abstract}

The lung is the organ in charge of gas exchange and ensuring good availability of oxygen in situations of maximum exercise such as the bull fight. For this reason, it s interesting to know which are the main pulmonary affectations seen in post-mortem carcasses in order to be able to make improvements. In the present work, the frequency of lung lesions has been studied for five years analyzing 251 fighting cat-

\footnotetext{
* Autor para correspondencia: aitor.fernandeznovo@uchceu.es
}

Cita del artículo: Fernandez-Novo A, Lomillos-Pérez JM, García-García JA (2020). Lesiones macroscópicas en pulmones en ganado de lidia. ITEA-Información Técnica Económica Agraria 116(2): 106-115. https://doi.org/10.12706/itea.2019.022 
tle, evaluating the presence of macroscopic lesions (emphysema, hemorrhage, congestion and bronchopneumonia) in the lungs of bovines of different ages and sexes. Methodology: it was carried out in the community of Madrid (July-September of the years 2013-2017). Results: $n=251$ animals in the five years, 87/251 presented lesions and anatomopathological changes. Among the lesions and postmortem changes observed: 22/87 correspond to pulmonary emphysema, 36/87 hemorrhage, 24/87 pulmonary congestion, 4/87 fibrinous bronchopneumonia and 1/87 suppurative bronchopneumonia. Steers (two-three year old males) presented a higher frequency of injuries (44.30 vs. $34.66 \% ; P=0.013$ ). The probability of suffering lung injury in steers, compared to bulls, turned out to be statistically significant, proving to be a protective factor the fact of being a bull (OR $=0.389 ; 95 \% \mathrm{Cl} ; 44.30$ vs. $23.63 \%)$. The occurrence of emphysema was more frequent in cows than in other type of animal $(53.33 \%$; $P=$ $0.023)$. Hemorrhage appeared less in cows than in other types of animals $(13.33 \% ; P=0.023)$, and tendency to appear less in bullfight calves $(P=0.023)$. The present study has revealed that two out of every three bovine breed cattle do not present macroscopic lung lesions, as well as that the most frequent lesion is pulmonary hemorrhage, followed by congestion and emphysema without observing high percentages of bronchopneumonia.

Taking into account that $94.25 \%$ of the observed findings correspond to agonizing lesions (presumably not corresponding to previous pathology, including pulmonary emphysema, bleeding and congestion), only $5.75 \%$ of the lesions would denote previous pathology. These results could reflect the conditions of extensity and maximum standards of animal welfare corresponding to the historical tradition of breeding wild cattle in pastures.

Keywords: Bull fight, emphysema, hemorrhage, bronchopneumonia, post-mortem.

\section{Introducción}

La raza de lidia representa un elevado porcentaje del número de animales en España, habiendo actualmente 206.385 cabezas de ganado en nuestro país, siendo la segunda raza en pureza después del ganado frisón (MAPA, 2019). Es una raza capaz de aprovechar gran variedad de terrenos, ocupar un elevado porcentaje de la dehesa española y perpetuar población en zonas más deshabitadas. Sus condiciones de manejo difieren del resto de la cabaña ganadera, pues viven de manera prácticamente silvestre, con poco contacto con el ser humano y en convivencia con la naturaleza (Edwards, 2010). De este modo, el índice de patologías por hacinamiento, contacto con otros rebaños y estrés es mínimo, no como sucede en otras poblaciones bovinas dedicadas a producción lechera o cárnica (Caucci et al., 2018).

No obstante, el destino final de la crianza de la raza de lidia se fundamenta en el espec- táculo taurino, que ha ido evolucionando a lo largo de la historia hasta tal punto que, en la actualidad, se exigen animales con mayor capacidad de rendimiento debido al alargamiento de la duración del espectáculo. La selección genética, la implantación de programas de manejo y la mejora en la nutrición han ayudado a conseguir aumentar estos rendimientos físicos de la cabaña de lidia muy notablemente (Cortés et al., 2008 y 2011; Lomillos et al., 2013).

De especial importancia es la perfecta funcionalidad pulmonar para lograr un adecuado estado físico para sobreponerse al ejercicio intenso del espectáculo. Por ello, el estudio de hallazgos patológicos pulmonares post-mortem (Wittum et al., 1996; Schneider et al., 2009) puede arrojar datos para orientar la mejora de la raza y del espectáculo. Conociendo la incidencia y prevalencia de las principales lesiones pulmonares, se podrían tomar medidas de prevención y mejora para maximizar la salud pulmonar. 
El pulmón bovino presenta diferencias anatómicas respecto a otras especies, con una marcada individualización de los lobulillos pulmonares por una mayor proporción de tejido conjuntivo en las paredes interlobulillares. Esto hace que el pulmón bovino sea más vulnerable a procesos inflamatorios y enfisema. Por otro lado, las neumonías son especialmente frecuentes en terneros de cebo. Tradicionalmente los procesos neumónicos en bovino se clasifican en bronconeumonía supurativa, bronconeumonía fibrinosa, neumonía intersticial o neumonitis, neumonía embolica y neumonía granulomatosa (López, 2011). En ganado de lidia estos patrones de lesiones pulmonares se documentan como hallazgos post-mortem muy infrecuentes, siendo los dos únicos representantes la neumonía supurativa y fibrinosa, ambas con un bajo porcentaje. Sin embargo, el esfuerzo que sufren pulmones previamente sanos, puede dar lugar a otro tipo de lesiones, especialmente frecuentes en este tipo específico de aptitud productiva, difícilmente extrapolables de lo descrito para el bovino de producción láctea o cárnica. Conocer cómo afecta el proceso de crianza, entrenamiento y mejora del toro en su hábitat específico, la dehesa, que difiere completamente de otros bovinos, y donde la información y el conocimiento científico son escasos, es de gran interés.

Por todo ello, se plantea la necesidad de estudiar, describir y discutir cuáles son los principales procesos patológicos pulmonares post-mortem que se aprecian en ganado de lidia después del espectáculo

\section{Objetivo}

El objetivo del presente estudio fue describir el alcance de las diferentes lesiones macroscópicas en pulmones observadas en desolladero después de la lidia en la Comunidad de Madrid durante los años 2013 a 2017 y relacionarla con los factores epidemiológicos recogidos.

\section{Material y métodos}

El estudio se realizó en la Comunidad Autónoma de Madrid, en plazas de toros con desolladero durante los meses de julio, agosto y septiembre, de los años 2013 a 2017. Los animales lidiados fueron vacas de suelta (hembras entre dos y doce años de edad), toros de capea (machos de tres años), toros de corrida de toros (machos entre cuatro y cinco años de edad) y novillos de novilladas picadas (machos de tres años) y no picadas (machos de dos años).

Todos los animales fueron supervisados por el mismo veterinario durante el desollado tras el sacrificio en el espectáculo taurino. Se realizó un análisis pormenorizado de canales, órganos y vísceras, con el objetivo de describir lesiones compatibles con decomiso de piezas, según establece legislación vigente en materia de salud pública (Orden 8345/1999, de 25 de noviembre del Consejo de Economía y Empleo; Real Decreto 260/2002, de 8 de marzo; Real Decreto 87/2014, de 24 de enero). Además, se examinaron detalladamente cada uno de los lóbulos pulmonares de cada animal, describiéndose las lesiones encontradas y recogiendo toda la información informáticamente. Se encuadró el tipo de lesión apreciada en el pulmón del animal en función de la siguiente clasificación (Sánchez-Pérez, 2012):

- Enfisema: acumulación patológica de aire el tejido pulmonar, debido a la dilatación de los alveolos o a la destrucción de sus paredes.

- Hemorragias: extravasación de sangre al parénquima o tejido pulmonar. Macroscópicamente la imagen varía desde pequeñas áreas de lesión (petequias) hasta lóbulos completos de color rojo intenso.

- Congestión pulmonar: acumulación de líquido en los pulmones, que da lugar a deterioro del intercambio gaseoso e hipoxemia arterial. Macroscópicamente, en la congestión aguda los pulmones presentan un aspecto similar a la hiperemia (color rojo oscuro). 
Por el tipo de sacrificio del ganado de lidia, ya bien por bala cautiva o estoque, el enfisema, las hemorragias y la congestión pulmonar se pueden producir como alteración post-mortem por muerte agónica (Fernández y Villalón, 1999; Pizarro et al., 2006).

- Bronconeumonía fibrinosa (Figura 1): lesión exudativa que comienza en la unión bronquiolo-alveolar y desde allí se extiende a bronquios y alveolos. El tipo de exudado es fibrinoso. Generalmente, localizada en lóbulos craneales (craneoventral) de uno o ambos pulmones, aunque en ocasiones puede extenderse hacia los principales. Lesión macroscópica: el aspecto varía en función de la evolución. En fases iniciales el pulmón posee una coloración rojiza intensa. Rápidamente la fibrina se deposita sobre la superficie pleural, y puede conferirle un aspecto de cristal esmerilado o formar placas de coloración amarillenta. Es frecuente observar un exudado fibrinoso en la cavidad pleural.

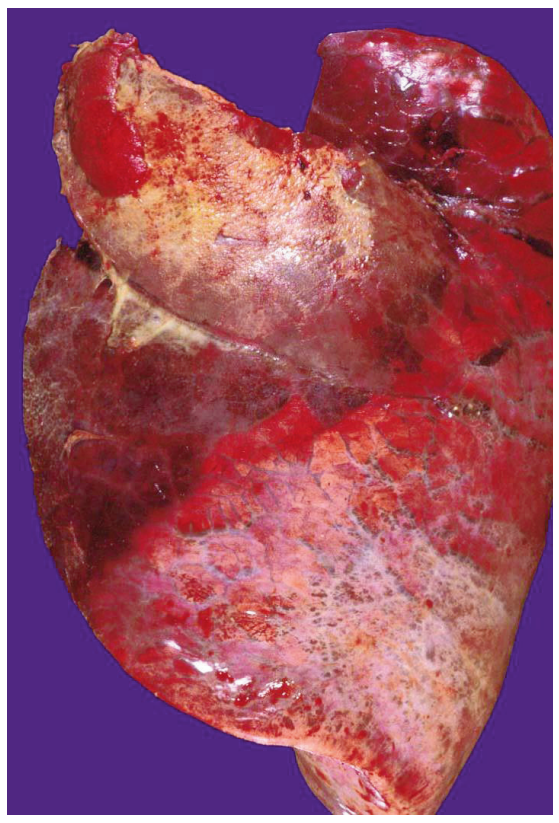

Figura 1. Pulmón con lesión de tipo bronconeumonía fibrinosa.

Figure 1. Lung with fibrinous bronchopneumonia lesion.
- Bronconeumonía supurativa (Figuras 2 y 3): lesión exudativa purulenta o mucopurulenta que comienza en la unión bronquiolo-alveolar y desde allí se extiende a bronquios y alveolos. La principal localización es en los lóbulos craneales (craneoventral) de uno o ambos pulmones. Las lesiones macroscópicas predominantes son: lobulillos consolidados, con una coloración que varía según progresa la lesión, de rojo intenso en fases tempranas (hiperemia y edema), a rosa grisáceo en fases instauradas (colapso de los alveolos por el exudado purulento) y gris pálido (semejante a carne de pescado fresco) en fases crónicas.

Se analizaron un total de 251 canales en diferentes salas autorizadas para el desollado de los animales tras el festejo (Real Decreto 260/2002, de 8 de marzo). De estas, 87/251 presentaron algún tipo de lesión verdadera o agónica.

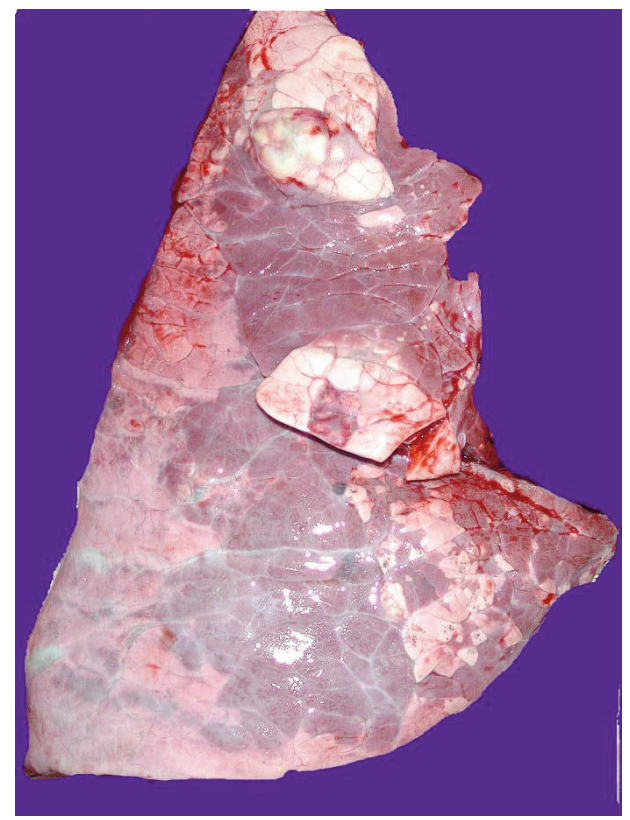

Figura 2. Pulmón con lesión de tipo bronconeumonía supurativa. Figure 2. Lung with supurative bronchopneumonia lesion. 


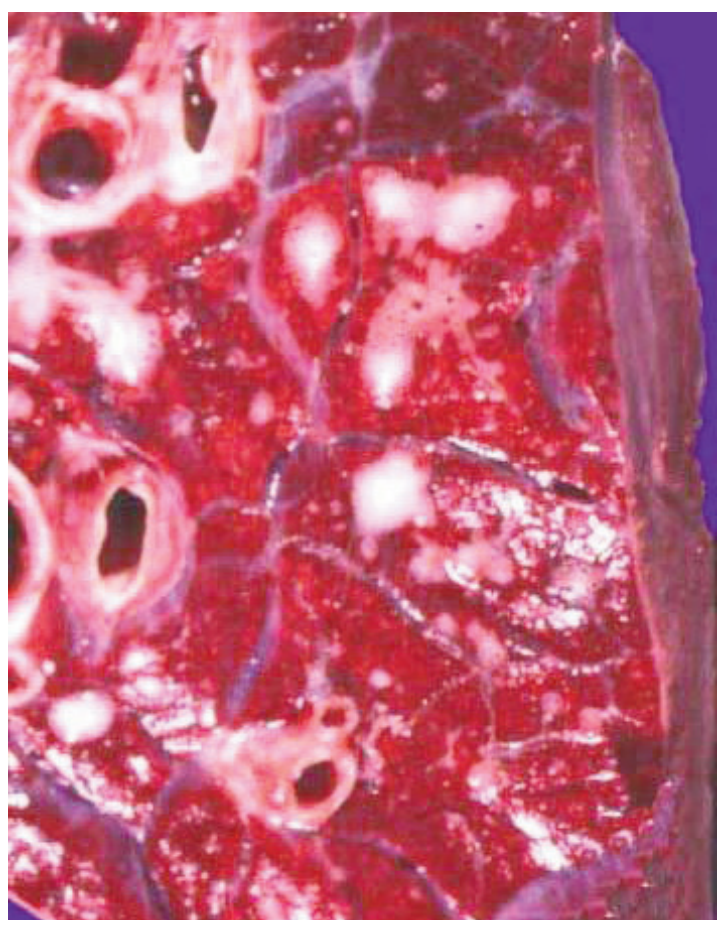

Figura 3. Sección transversal de lóbulo pulmonar con lesión de tipo bronconeumonía supurativa. Figure 3. Transverse section of pulmonary lobe with suppurative bronchopneumonia lesion.

La información se procesó estadísticamente con IBM-SPSS ${ }^{\circledR}$ V25.0 (IBM, Armonk, New York, USA), mediante la prueba de chi-cuadrado y análisis de regresión logística.

\section{Resultados}

Los resultados obtenidos tras el estudio se recogen en las tablas 1 (categoría de animal, presencia de lesión y año de estudio) y 2 (categoría de animal y tipo de lesión o alteración agónica -post-mortem-).

La frecuencia de las lesiones y hallazgos patológicos observados por cada año no presentó diferencias estadísticamente significativas $(P=0,364)$, si bien por tipo de animal, los to- ros (bovinos machos mayores de cuatro años de edad usados en corridas de toros) sí presentaron significativamente una menor frecuencia de lesiones que el resto $(23,63$ vs. $34,66 \% ; P=0,013$ ) y los novillos (machos de dos a tres años lidiados en novilladas), fueron los animales que presentaron mayor frecuencia de lesiones verdaderas y agónicas (44,30 vs. $34,66 \% ; P=0,013)$. La frecuencia de alteraciones agónicas -post-mortem-fue del $36,27 \%(82 / 251)$ sobre el total de los animales, mientras que del $94,25 \%$ (82/87) sobre el total de hallazgos anatomopatológicos. Se consideraron el conjunto de lesiones halladas en desolladero, tanto lesiones agónicas como reales. El análisis una vez descartadas las lesiones agónicas como no lesión verdadera, suponía la pérdida de potencia estadística, ya que la cantidad de pulmones con lesiones patológicas reales era reducida $(P>0,5)$.

La probabilidad de padecer algún tipo de lesión o alteración post-mortem pulmonar en novillos, respecto a los toros, resultó ser estadísticamente significativa, siendo los animales de mayor edad (los toros de 4 y 5 años) los que menores lesiones presentaron, reduciéndose en ellos un $60 \%$, aproximadamente (OR =0,389; Cl 95\%, 44,30 vs. $23,63 \%$ ). Las otras dos categorías (novillos vs. vaca y toro de capea, OR =0,992 y OR =0,883; Cl $95 \%$, 44,30 vs. $44,11 \% ; 44,30$ vs. $39,28 \%$, respectivamente), comparadas con novillos, no mostraron diferencias significativas $(P>0,5)$.

No se observaron diferencias estadísticamente significativas entre la aparición de un tipo de alteración agónica -post-mortem-o lesión en función del año $(P=0,714)$.

La aparición de enfisema fue más frecuente en vacas que en otros tipos de animales, con una afectación media del $53,33 \%$ de los individuos en el periodo estudiado $(P=0,023)$. Sin embargo, existe una tendencia a aparecer menos lesiones enfisematosas en novillos que en otro tipo de animal de lidia, con un alcance del $14,28 \%$ de los individuos en el periodo estudiado $(P=0,023)$. 
Tabla 1. Relación de animales analizados con presencia de lesión o alteración post-mortem por categoría de animal y año.

Table 1. List of animals analyzed with presence of injury or post-mortem changes by animal category and year.

\begin{tabular}{lccccccc}
\hline Tipo de animales & $\mathrm{n}$ & 2013 & 2014 & 2015 & 2016 & 2017 & Totales \\
\hline Vacas suelta & 34 & $\begin{array}{c}16,66 \% \\
(1 / 6)\end{array}$ & $\begin{array}{c}50,00 \% \\
(4 / 8)\end{array}$ & $\begin{array}{c}50,00 \% \\
(4 / 8)\end{array}$ & $\begin{array}{c}42,85 \% \\
(3 / 7)\end{array}$ & $\begin{array}{c}60,00 \% \\
(3 / 5)\end{array}$ & $\begin{array}{c}44,11 \% \\
(15 / 34)^{\mathrm{e}}\end{array}$ \\
& & & & & & \\
Toros capea & 28 & $33,33 \%$ & $16,66 \%$ & $42,85 \%$ & $60,00 \%$ & $50,00 \%$ & $39,28 \%$ \\
& & $(2 / 6)$ & $(1 / 6)$ & $(3 / 7)$ & $(3 / 5)$ & $(2 / 4)$ & $(11 / 28)^{\mathrm{d}}$ \\
Toros & 110 & $17,39 \%$ & $22,72 \%$ & $21,73 \%$ & $27,27 \%$ & $30,00 \%$ & $23,63 \%$ \\
& & $(4 / 23)$ & $(5 / 22)$ & $(5 / 23)$ & $(6 / 22)$ & $(6 / 20)$ & $(26 / 110)^{\mathrm{c}}$ \\
Novillos & 79 & $37,50 \%$ & $38,88 \%$ & $40,00 \%$ & $56,25 \%$ & $50,00 \%$ & $44,30 \%$ \\
& & $(6 / 16)$ & $(7 / 18)$ & $(6 / 15)$ & $(9 / 16)$ & $(7 / 14)$ & $(35 / 79)$ \\
Totales & 251 & $25,49 \%$ & $31,49 \%$ & $33,96 \%$ & $42,00 \%$ & $41,86 \%$ & $34,66 \%$ \\
& & $(13 / 51)^{\mathrm{a}}$ & $(17 / 54)^{\mathrm{a}}$ & $(18 / 53)^{\mathrm{a}}$ & $(21 / 50)^{\mathrm{a}}$ & $(18 / 43)^{\mathrm{a}}$ & $(87 / 251)^{\mathrm{a}}$ \\
\hline
\end{tabular}

Significación estadística: a $P=0,364 ;{ }^{b} P=0,013 ;{ }^{c} \mathrm{OR}=0,389(\mathrm{Cl} 95 \%, 44,30$ vs. 23,63\%); $\mathrm{d}$ OR $=0,883$ (Cl 95\%, 44,30 vs. 39,28\%); e $\mathrm{OR}=0,992$ (Cl 95\%, 44,30 vs. 44,11\%).

Tabla 2. Relación de animales analizados por categoría de animal y tipo de alteración agónica -postmortem- * o lesión patológica, macroscópica.

Table 2. List of animals analyzed by animal category and type of post-mortem changes or macroscopic lesion.

\begin{tabular}{|c|c|c|c|c|c|c|}
\hline & & $\begin{array}{l}\text { Vacas } \\
\text { suelta }\end{array}$ & $\begin{array}{l}\text { Toros } \\
\text { capea }\end{array}$ & Toros & Novillos & Totales \\
\hline \multirow[t]{3}{*}{ Totales } & $\begin{array}{l}\text { Animales con alt. agónica / } \\
\text { total animales }\end{array}$ & $\begin{array}{r}41,17 \% \\
(14 / 34)\end{array}$ & $\begin{array}{c}32,14 \% \\
(9 / 28)\end{array}$ & $\begin{array}{l}22,72 \% \\
(25 / 110)\end{array}$ & $\begin{array}{c}43,03 \% \\
(34 / 79)\end{array}$ & $\begin{array}{l}32,67 \% \\
(82 / 251)\end{array}$ \\
\hline & $\begin{array}{l}\text { Animales con lesión / } \\
\text { total animales }\end{array}$ & $\begin{array}{l}2,94 \% \\
(1 / 34)\end{array}$ & $\begin{array}{l}7,14 \% \\
(2 / 28)\end{array}$ & $\begin{array}{l}0,90 \% \\
(1 / 110)\end{array}$ & $\begin{array}{l}1,26 \% \\
(1 / 79)\end{array}$ & $\begin{array}{l}1,99 \% \\
(5 / 251)\end{array}$ \\
\hline & $\begin{array}{l}\text { Animales con lesión y alt. } \\
\text { agónica / total animales }\end{array}$ & $\begin{array}{c}44,11 \% \\
(15 / 34)\end{array}$ & $\begin{array}{l}39,28 \% \\
(11 / 28)\end{array}$ & $\begin{array}{l}23,63 \% \\
(26 / 110)\end{array}$ & $\begin{array}{c}44,30 \% \\
(35 / 79)\end{array}$ & $\begin{array}{l}34,66 \% \\
(87 / 251)\end{array}$ \\
\hline \multirow[t]{3}{*}{$\begin{array}{l}\text { Tipo de alteración } \\
\text { agónica }\end{array}$} & Enfisema & $\begin{array}{l}53,33 \% \\
(8 / 15)^{a}\end{array}$ & $\begin{array}{l}45,45 \% \\
(5 / 11)\end{array}$ & $\begin{array}{c}15,38 \% \\
(4 / 26)\end{array}$ & $\begin{array}{l}14,28 \% \\
(5 / 35)^{a}\end{array}$ & $\begin{array}{l}25,28 \% \\
(22 / 87)\end{array}$ \\
\hline & Hemorragia & $\begin{array}{l}13,33 \% \\
(2 / 15)^{a}\end{array}$ & $\begin{array}{l}18,18 \% \\
(2 / 11)^{a}\end{array}$ & $\begin{array}{l}53,84 \% \\
(14 / 26)\end{array}$ & $\begin{array}{l}51,42 \% \\
(18 / 35)\end{array}$ & $\begin{array}{c}41,37 \% \\
(36 / 87)\end{array}$ \\
\hline & Congestión & $\begin{array}{c}26,66 \% \\
(4 / 15)\end{array}$ & $\begin{array}{c}18,18 \% \\
(2 / 11)\end{array}$ & $\begin{array}{c}26,92 \% \\
(7 / 26)\end{array}$ & $\begin{array}{l}31,42 \% \\
(11 / 35)\end{array}$ & $\begin{array}{c}27,58 \% \\
(24 / 87)\end{array}$ \\
\hline \multirow[t]{2}{*}{$\begin{array}{l}\text { Tipo de lesión } \\
\text { patológica }\end{array}$} & Bronconeumonía fibrinosa & $\begin{array}{l}6,66 \% \\
(1 / 15)\end{array}$ & $\begin{array}{l}9,09 \% \\
(1 / 11)\end{array}$ & $\begin{array}{c}3,84 \% \\
(1 / 26)\end{array}$ & $\begin{array}{c}2,85 \% \\
(1 / 35)\end{array}$ & $\begin{array}{c}4,59 \% \\
(4 / 87)\end{array}$ \\
\hline & Bronconeumonía supurativa & $\begin{array}{l}0,00 \% \\
(0 / 15)\end{array}$ & $\begin{array}{l}9,09 \% \\
(1 / 11)^{a}\end{array}$ & $\begin{array}{c}0,00 \% \\
(0 / 26)\end{array}$ & $\begin{array}{c}0,00 \% \\
(0 / 35)\end{array}$ & $\begin{array}{l}1,14 \% \\
(1 / 87)\end{array}$ \\
\hline
\end{tabular}

*Se considera alteración agónica: enfisema, hemorragia y congestión pulmonar. Significación estadística: a $P=0,023$. 
La hemorragia fue observada menos en vacas que en otro tipo de animal, con una repercusión del $13,33 \%$ en el periodo estudiado $(P$ $=0,023$ ). Igualmente se demostró una tendencia a que apareciera menos en toro de capea $(P=0,023)$.

La bronconeumonía supurativa apareció más en toro de capea que en otro tipo de animal de lidia, con una frecuencia de aparición del $9,09 \%$ en el periodo estudiado $(P=0,023)$.

\section{Discusión}

El presente estudio ha revelado que dos de cada tres bóvidos de raza de lidia no presentan lesiones macroscópicas pulmonares, así como que la lesión más incidente es la hemorragia pulmonar, seguida de congestión.

Por otra parte, prácticamente no se observan casos de bronconeumonías (4,59\% fibrinosa y $1,14 \%$ supurativa). Estos resultados podrían reflejar las condiciones de extensividad y máximos estándares de bienestar animal correspondientes a la tradición histórica de crianza del ganado bravo en dehesas.

Los factores limitantes del toro durante la lidia se pueden resumir en disminución de la volemia y número de glóbulos rojos, lesión muscular, deshidratación, fatiga, acidosis y fuerte depleción del glucógeno muscular, entre otros (Agüera Buendía y Requena Domenech, 2011). Esto hizo pensar a los ganaderos en entrenar al toro bravo antes del espectáculo como método de aumento de su eficiencia en plaza, no obstante, la poca bibliografía existente arroja resultados dispares (Escribano et al., 2010; Requena, 2012). Escalera-Valente et al. (2008) por su parte, estudiaron el efecto de la lidia sobre los gases sanguíneos, cuyas concentraciones reflejaron una inadaptación del animal al ejercicio llevado a cabo en el ruedo, aun habiendo sido entrenados físicamente antes del festejo. A su vez, el estudio de Martínez-Gomariz et al. (1999) puso de manifiesto algunas lesiones musculares acompañadas de alteraciones del tejido conectivo del toro tras la lidia.

En nuestro estudio, la aparición de lesiones pulmonares de cualquier tipo (incluidas las agónicas) se situó en el 34,66\% de las 251 canales analizadas. Comparativamente este dato es menor a los obtenidos en otros trabajos recientes (Caucci et al., 2018) en vacuno de carne, en los que describieron tasas del $64 \%$ de uno o ambos pulmones con algún tipo de afección o lesión. Además, si consideramos lesiones agónicas las de tipo hemorrágico, congestivo y enfisematoso, se reduciría el porcentaje de lesiones reales al 5,75\% de los individuos. Esto podría reflejar el impacto positivo que tiene el hábitat durante toda la vida productiva de esta raza en comparación con otras razas cárnicas. Aunque el estudio previamente citado (Caucci et al., 2018) reveló que la presencia de lesiones pulmonares no está directamente relacionada con deterioros en la calidad de la canal de los animales, ésta sí repercute de manera negativa en los índices de conversión así como en la rentabilidad por $\mathrm{kg}$ de canal, pues lesiones pulmonares aumentan los índices de conversión, y por ende, empeoran el rendimiento económico (Thompson et al., 2006).

La aparición de enfisema en nuestro estudio, es más frecuente en vacas que en los otros tipos de animales de lidia (53,33\% vs. $25,28 \%$; $P=0,023)$. Lo podemos atribuir a que estos animales, de conformidad con la Orden 8345/ 1999, deben ser sometidos a procedimientos de aturdimiento mecánico previo al sacrificio. Gomes et al. (1999) demostraron que el enfisema intersticial se produce por una inspiración forzada observado en aquellos bovinos aturdidos de manera incorrecta; esta lesión se denomina enfisema agónico (Ríos et al., 2012). Porcentajes mayores de incidencia de lesiones enfisematosas se han descrito en algunos mataderos, pudiendo llegar al $60 \%$ (Oviedo-Socarrás et al., 2016). 
El bajo número de lesiones hemorrágicas registradas en vaca y toro de capea, en comparación con el resto de animales, puede estar causado por el tipo de sacrificio que se realiza con cada animal, pues vacas y toros de capea, según legislación vigente se sacrifican con bala cautiva, mientras que novillos y toros, dedicados a novilladas y corridas de toros, respectivamente, se sacrifican con estoque. Por ende, aparecen con mayor frecuencia lesiones hemorrágicas en pulmón en estos animales (Pizarro et al., 2006).

Las bronconeumonías supurativas del ganado bovino están producidas principalmente por Pasteurella multocida, Actinomyces pyogenes o Mycoplasma bovis y las bronconeumonías fibrinosas por Mannhemia haemolytica, Mycoplasma mycoides y Histophylussommi (Bagnis et al., 2006). En cebaderos de Canadá se observó que entre el $41-45 \%$ de neumonías fibrinosas se ocasionan por Mannhemia haemolytica (Radostits, 2007). En nuestro trabajo, sin haber realizado un estudio microbiológico, se aprecia una baja frecuencia de aparición en ambos tipos de bronconeumonía (supurativa 1,14 y fibrinosa 4,59\%), con lo que entendemos un bajo nivel de infección por estos agentes, probablemente debido al hábitat y sistema de manejo extensivo que ocupan todos los animales de la raza de lidia. El medio en el que viven (ecosistema de dehesa, en total convivencia con fauna silvestre y entorno natural) les previene de los factores predisponentes de las neumonías (Edwards, 2010), como estrés, falta de higiene y hacinamiento, inclemencias climatológicas, alimentación, etc. En vacuno lechero (González-Martín y Pérez-Villalobos, 2015) describen morbilidades de neumonías desde el 3,3\% hasta el $20 \%$; si bien, estas prevalencias son mayores a las observadas por nosotros debido, probablemente, a las condiciones de intensividad del ganado vacuno lechero. En vacuno de cebo se describen diferentes prevalencias en función de las con- diciones de manejo, metafilaxia, prevención vacunal, hacinamiento, instalaciones y alimentación (Edwards, 2010). Además, se debe tener en cuenta el momento del sacrificio para consumo, pues en cebaderos está entorno a doce - catorce meses en su mayoría, sin embargo, en ganado bravo depende del tipo de festejo taurino al que se vaya a dedicar esa res (3-4 años novillos, 4-6 años toros y vacas de suelta de todas las edades). Thompson et al. (2006) describen una repercusión del $29,7 \%$ de lesiones pulmonares en animales con patologías pulmonares subclínicas, y del $22,6 \%$ en animales ya con cuadro clínico. Leruste et al. (2012) obtiene valores máximos de afectación del $28 \%$ de los animales en cebo a las tres semanas de edad, mientras que al final del cebo señala valores máximos del $39 \%$ de animales con sintomatología pulmonar. Dichos datos contrastan con los obtenidos en el estudio, en animales de mayor edad, donde fueron registrados menos casos ( 4,59 y $1,14 \%$ respectivamente), siendo los animales de capea los que presentaron un mayor porcentaje, ya que con este tipo de animales no se tiene un manejo sanitario tan cuidado como en los novillos y toros destinados a festejos mayores (corridas y novilladas). La principal hipótesis de esta disparidad de datos está en las condiciones de manejo y edades de sacrificio: el ganado de lidia vive en condiciones de extensividad, frente a la intensificación de los cebaderos; además, los tiempos de sacrificio son muy dispares, como hemos comentado anteriormente.

Finalmente, cabe destacar la baja aparición de lesiones pulmonares halladas tras este estudio, aun habiendo incorporado a la estadística lesiones que se pueden considerar como alteraciones post-mortem. Por lo tanto, la frecuencia real sería aún más baja de lo descrito, 5,75\%, lo cual apoya los máximos estándares de bienestar animal, manejo y hábitat característico en gran parte de las explotaciones de ganado de lidia en nuestro país. Por ello, se 
debe promocionar el entorno y manejo al que están sometidos estos animales, pues se corresponde con unas excelentes condiciones de productividad.

Futuros estudios incluyendo datos de edad, encaste, situación geográfica de la explotación, así como de otras variables, ayudarán a definir aún más la frecuencia de lesiones pulmonares y su etiología.

\section{Conclusiones}

La frecuencia de aparición de lesiones pulmonares en ganado bovino de lidia es inferior a la descrita por otros autores en vacuno lechero, vacuno de carne adulto en extensivo y vacuno de cebo.

El tipo de lesiones se mantiene con poca variación a lo largo de los años estudiados.

\section{Agradecimientos}

Gracias a todos los ganaderos, compañeros veterinarios y empresarios taurinos por las facilidades en la toma de datos durante todos estos años.

La elaboración de este estudio se ha realizado sin financiación de ningún ente, ni organismo público, ni privado.

\section{Referencias bibliográficas}

Agüera Buendía E, Requena Domenech F (2011). Factores limitantes del rendimiento físico del toro bravo durante la lidia. Anales Real Academia de Ciencias Veterinarias de Andalucía Orienta 24(1): 193-211.

Bagnis G, Rabaglino MB, Raviolo JM, Schleef N (2006). Estudio histopatológico, etiológico e inmunohistoquímico de lesiones compatibles con neumonías intersticiales en el ganado bovino. Revista Electrónica de Veterinaria 7(9): 1-7.

Caucci C, Di Martino G, Schiavon E, Garbo A, Soranzo E, Tripepi L, Stefani AL, Gagliazzo L, Bonfanti $L$ (2018). Impact of bovine respiratory disease on lung lesions, slaughter performance and antimicrobial usage in French beef cattle finished in North-Eastern Italy. Italian Journal of Animal Science 17(4): 1065-1069. https://doi. org/10.1080/1828051X.2018.1426395

Cortés O, Tupac-Yupanqui I, Dunner S, GarcíaAtance MA, García D, Fernández J, Cañón J (2008). Ancestral matrilineages and mitochondrial DNA diversity of the Lidia cattle breed. Animal Genetics 39(6): 649-654. https://doi.org/ 10.1111/j.1365-2052.2008.01782.x

Cortés $\mathrm{O}$, Tupac-Yupanqui I, Dunner S, Fernández J, Cañón J (2011). Y chromosome genetic diversity in the Lidia bovine breed: a highly fragmented population. Journal of Animal Breeding and Genetics 128(6): 491-496. https://doi. org/10.1111/j.1439-0388.2011.00951.x

Edwards TA (2010). Control methods for bovine respiratory disease for feedlot cattle. Veterinary Clinics: Food Animal Practice 26(2): 273284. https://doi.org/10.1016/j.cvfa.2010.03.005

Escalera-Valente F, González-Montaña JR, Alonso de la Varga ME, Lomillos-Pérez JM, Gaudioso-Lacasa VR (2013). Influence of intense exercise on acid-base, blood gas and electrolyte status in bulls. Research in Veterinary Science 95; 623-628. http://dx.doi.org/10.1016/j.rvsc.2013.03.018

Escribano B, Tunez I, Requena F, Rubio M, De Miguel R, Montilla P, Tovar P, Aguera E (2010). Effects of an aerobic training program on oxidative stress biomarkers in Bulls. Veterinarni Medicina 55(9): 422-428. https://doi.org/10. 17221/2979-VETMED

Fernández J, Villalón J (1999). Estudio de las lesiones producidas por suerte de varas en la segunda parte de la feria de San Isidro 1998. Revista de estudios taurinos 9: 113-140.

Gomes NBN, Rostagno MH, Santos GJVG, Aguiar PHP (1999). Frecuencia de las lesiones en bovinos en el matadero municipal de la ciudad de Labras, MG. Veterinaria noticias 5(1): 41-46. 
González-Martín JV, Pérez-Villalobos N (2015). Etiopatogenia. En: Síndrome Respiratorio Bovino. pp. 24-28. Edit. Servet.

Leruste $\mathrm{H}$, Brscic M, Heutinck LFM, Visser EK, Wolthuis-Fillerup M, Bokkers EAM, Stockhofe-Zurwieden N, Cozzi G, Gottardo F, Lensink BJ, van Reenen CG (2012). The relationship between clinical signs of respiratory system disorders and lung lesions at slaughter in veal calves. Preventive Veterinary Medicine 105(1-2): 93-100. https:// doi.org/10.1016/j.prevetmed.2012.01.015

Lomillos JM, Alonso ME y Gaudioso V (2013). Análisis de la evolución del manejo en las explotaciones de toro de lidia. Desafíos del sector. ITEA-Información Técnica Económica Agraria 109(1): 49-68.

López A (2011). Respiratory System, Mediastinum and Pleurae. En: Pathological Basis of Veterinary Disease, 5th Edition. (Eds Zachary J, Mc Gavin M) pp. 458-538. Eds. Elsevier.

MAPA (2019). Raza bovina LIDIA, Catálogo oficial de razas. Ministerio de Agricultura, Pesca y Alimentación. Disponible en: https://www.mapa. gob.es/es/ganaderia/temas/zootecnia/razas-ganaderas/razas/catalogo/autoctona-fomento/bovino/lidia/iframe-ejemplo-arca.aspx (Consultado: 15 abril 2019).

Martínez-Gomariz F, Vázquez JM, Gil F, Moreno F, Ramírez G, Latorre R, López Albors O (1999). Lesiones musculares en el toro bravo (Bos Taurus ibericus) después de la lidia. Anales de Veterinaria de Murcia 15: 17-24.

Oviedo-Socarrás T, Vargas-Viloria M, Cardona JA (2016). Frecuencia de lesiones macroscópicas en pulmones de bovinos tipo explotación faenados en el frigorífico del municipio de Montería, Colombia. Revista de la Facultad de Medicina Veterinaria y de Zootecnia 63(1): 30-38.

Pizarro M, Castaño M, Mazzuchelli F, García IR, Parrilla G (2006). Significación sanitaria de las lesiones más frecuentes en el desolladero. Calidad y características de la carne de lidia. Bovis 131: 59-71.
Radostits OM, Gay CC, Hinchcliff KW, Constable PD (2007). Diseases associated with Pasteurella species. En: Veterinary Medicine: A textbook of the diseases of cattle, horses, sheep, pigs and goats. $10^{\mathrm{a}}$ ed. pp. 921-962. Saunders Elsevier.

Requena F (2012). Evaluación de la capacidad física tras la lidia con el entrenamiento. Tesis doctoral. Universidad de Córdoba.

Ríos FG, Estrada A, Hernández J, Pérez C, Portillo JJ, Robles JC (2012). Factores que influyen en la emesis post-aturdimiento en bovinos. Revista mexicana de ciencias pecuarias 3(3): 343-356.

Sánchez-Pérez MA (2012). Anatomía patológica del aparato respiratorio. Cátedra de anatomía patológica de la Universidad Complutense de Madrid.

Schneider MJ, Tait RG, Busby WD, Reecy JM (2009). An evaluation of bovine respiratory disease complex in feedlot cattle: Impact on performance and carcass traits using treatment records and lung lesion scores. Journal of Animal Science 87(5): 1821-1827. https://doi.org/10. 2527/jas.2008-1283

Thompson PN, Stone A, Schultheiss WA (2006). Use of treatment records and lung lesion scoring to estimate the effect of respiratory disease on growth during early and late finishing periods in South African feedlot cattle. Journal of Animal Science 84(2): 488-498. https://doi. org/10.2527/2006.842488x

Wittum TE, Woollen NE, Perino LJ, Littledike ET (1996). Relationships among treatment for respiratory tract disease, pulmonary lesions evident at slaughter and rate of weight gain in feedlot cattle. Journal of the American Veterinary Medical Association 209(4): 814-818.

(Aceptado para publicación el 14 de octubre de 2019) 